\title{
Listeria monocytogenes Associated with Pasteurized Chocolate Milk, Ontario, Canada
}

\author{
Heather Hanson, Yvonne Whitfield, Christina Lee, \\ Tina Badiani, Carolyn Minielly, Jillian Fenik, \\ Tony Makrostergios, Christine Kopko, \\ Anna Majury, Elizabeth Hillyer, Lisa Fortuna, \\ Anne Maki, Allana Murphy, Marina Lombos, \\ Sandra Zittermann, Yang Yu, Kristin Hill, \\ Adrienne Kong, Davendra Sharma, \\ Bryna Warshawsky
}

In an investigation of a listeriosis outbreak in Ontario, Canada, during November 2015-June 2016, pasteurized chocolate milk was identified as the source. Because listeriosis outbreaks associated with pasteurized milk are rare in North America, these findings highlight that dairy products can be contaminated after pasteurization.

$L$ isteria monocytogenes is a formidable pathogen acquired primarily through contaminated food. Invasive listeriosis is a reportable disease in Ontario, Canada; $\approx 50$ case-patients ( 0.4 cases $/ 100,000$ persons) have been reported annually since 2005 (1). Recent outbreaks of listeriosis in North America have been associated with delicatessen meats, soft cheeses, raw produce, and unpasteurized dairy products $(2-4)$. However, listeriosis outbreaks linked to pasteurized fluid milk are rare.

A study in the United States reviewed 83 fluid milkassociated disease outbreaks during 1990-2006; however, only 1 outbreak was attributed to $L$. monocytogenes (5). We report an outbreak of listeriosis associated with pasteurized chocolate milk in Ontario, Canada.

\section{The Study}

We defined an outbreak case-patient as a person in Ontario with listeriosis symptom onset after November 1, 2015,

Author affiliations: Public Health Ontario, Toronto, Ontario, Canada (H. Hanson, Y. Whitfield, C. Lee, T. Badiani, A. Majury, L. Fortuna,

A. Maki, A. Murphy, M. Lombos, S. Zittermann, Y. Yu,

B. Warshawsky); Simcoe Muskoka District Health Unit, Barrie,

Ontario, Canada (C. Minielly, J. Fenik, T. Makrostergios);

Canadian Food Inspection Agency, Ottawa, Ontario, Canada

(C. Kopko, K. Hill, A. Kong, D. Sharma); Public Health Agency of

Canada, Guelph, Ontario, Canada (E. Hillyer); Western University,

London, Ontario, Canada (B. Warshawsky)

DOI: https://doi.org/10.3201/eid2503.180742 who had pulsed-field gel electrophoresis (PFGE) pattern combinations LMACI.0015/LMAAI.0024 or LMACI.0015/LMAAI.0069. Thirty-four case-patients met the outbreak definition; only Ontario residents were identified. Eleven case-patients had an onset date during November 14, 2015-February 14, 2016. Onset dates ranged from April 11 to June 20, 2016, for 21 case-patients in the second wave; the remaining 2 case-patients were outliers (Figure 1). Median age was 73 years (range $<1-90$ years). More than half of the case-patients were female $(20 / 34,59 \%)$. Hospitalizations occurred for 32 (94\%) case-patients, and 4 deaths $(12 \%)$ were reported.

In Ontario, local public health professionals complete the national invasive listeriosis questionnaire and collect food samples. We conducted a case-case analysis by using Ontario case-patients listed in the national listeriosis database as controls. We used a variety of methods to support hypothesis generation, including supplemental questionnaires, centralized interviewing, and reviewing purchase records collected through shoppers' loyalty card programs. A meeting was also held with representatives from a grocery chain that was common for case-patients (retail chain A) for insights into possible sources.

PFGE and whole-genome sequencing were performed at the Public Health Ontario Laboratory, in accordance with PulseNet Canada protocols (Table; Appendix Figure, https:// wwwnc.cdc.gov/EID/article/25/3/18-0742-App1.pdf). Food safety investigations, including targeted retail sampling, were conducted by the Canadian Food Inspection Agency and Ontario Ministry of Agriculture, Food and Rural Affairs. Laboratory analyses of food samples were conducted by the Canadian Food Inspection Agency and the Public Health Ontario Laboratory.

Several hypotheses were generated during the course of this outbreak. In the first wave, a concurrent listeriosis outbreak associated with leafy greens was ongoing in the United States and Canada. However, product testing did not establish a relationship between the 2 outbreaks. Cheddar cheese was also suspected, but a food safety investigation, including sampling at the manufacturer, did not support a link to this outbreak $(6,7)$. Although leafy greens and cheddar cheese were ruled out, 1 commonality remained; shopping at retail chain A was reported frequently by case-patients. 
Table. Characteristics of 23 Listeria monocytogenes isolates analyzed by whole-genome sequencing during listeriosis outbreak in pasteurized chocolate milk, Ontario, Canada*

\begin{tabular}{|c|c|c|c|}
\hline Isolate ID & Isolate source & PFGE pattern, first enzyme/second enzyme & SRA accession no. \\
\hline ON-1501 & Human & LMACI.0015/LMAAI.0069 & SAMN09909078 \\
\hline ON-1502 & Human & LMACI.0015/LMAAI.0024 & SAMN09909079 \\
\hline ON-1503 & Human & LMACI.0015/LMAAI.0024 & SAMN09909080 \\
\hline ON-1601 & Human & LMACI.0015/LMAAI.0069 & SAMN09909081 \\
\hline ON-1602 & Human & LMACI.0015/LMAAI.0024 & SAMN09909082 \\
\hline ON-1603 & Human & LMACI.0015/LMAAI.0069 & SAMN09909083 \\
\hline ON-1604 & Human & LMACI.0015/LMAAI.0024 & SAMN09909084 \\
\hline ON-1605 & Human & LMACI.0015/LMAAI.0069 & SAMN09909085 \\
\hline ON-1606 & Human & LMACI.0015/LMAAI.0069 & SAMN09909086 \\
\hline ON-1607 & Human & LMACI.0015/LMAAI.0069 & SAMN09909087 \\
\hline ON-1608 & Human & LMACI.0015/LMAAI.0024 & SAMN09909088 \\
\hline ON-1609 & Human & LMACI.0015/LMAAI.0069 & SAMN09909089 \\
\hline ON-1610 & Human & LMACI.0015/LMAAI.0024 & SAMN09909090 \\
\hline ON-1611 & Human & LMACI.0015/LMAAI.0069 & SAMN09909091 \\
\hline ON-1612 & Human & LMACI.0015/LMAAI.0024 & SAMN09909092 \\
\hline ON-1613 & Human & LMACI.0015/LMAAI.0069 & SAMN09909093 \\
\hline ON-1614 & Human & LMACI.0015/LMAAI.0069 & SAMN09909094 \\
\hline ON-1615 & Human & LMACI.0015/LMAAI.0069 & SAMN09909095 \\
\hline ON-1616 & Human & LMACI.0015/LMAAI.0069 & SAMN09909096 \\
\hline ON-1617 & Human & LMACI.0015/LMAAI.0069 & SAMN09909097 \\
\hline ON-1618 & Human & LMACI.0015/LMAAI.0069 & SAMN09909098 \\
\hline ON-1619 & Human & LMACI.0015/LMAAI.0069 & SAMN09909099 \\
\hline ON-1620 & Human & LMACI.0015/LMAAI.0069 & SAMN09909100 \\
\hline ON-1621 & Human & LMACI.0015/LMAAI.0069 & SAMN09909101 \\
\hline ON-1622 & Human & LMACI.0015/LMAAI.0069 & SAMN09909102 \\
\hline ON-1623 & Chocolate milk & LMACI.0015/LMAAI.0069 & SAMN09909103 \\
\hline
\end{tabular}

A second wave began in April 2016 in which 10 of 17 case-patients reported consuming coleslaw. Six casepatients ate coleslaw from the same manufacturer, which supplied retail chain A and a fast food restaurant chain. However, the food safety investigation, including sampling at the manufacuturer and supplier, did not support this hypothesis.

On May 24, 2016, L. monocytogenes isolated from expired bagged chocolate milk collected from the home of 1 case-patient was confirmed to have the outbreak strain
PFGE pattern. Fluid milk in Canada is often sold in plastic bags (Figure 2). In this instance, the outer packaging, which is the only area that contains the brand name, was discarded. Thus, the brand name was uncertain, and efforts were undertaken to confirm the source of the chocolate milk. Because the proxy of the case-patient reported purchasing brand B milk, samples of brand B chocolate and white milk were collected from retail for testing. Brand B was the main brand of chocolate milk sold by retail chain A, and it is distributed primarily in Ontario.
Figure 1. Outbreak cases of listeriosis $(\mathrm{n}=$ 34 ) by onset week and year, Ontario, Canada, November 2015-August 2016. Data were obtained from the Ontario Ministry of Health and Long-Term Care, integrated Public Health Information System database, extracted by Public Health Ontario, August 16, 2016. Weeks are defined according to the Public Health Agency of Canada epidemiologic week calendar. *Neonatal case-patient with symptom onset on April 4, 2016 (week 14), and illness most likely caused by mother-to-child transmission. **Asymptomatic case-patient from whom a specimen was collected on July 13,2016 , and exposure occurred before June 27, 2016 (week 28).

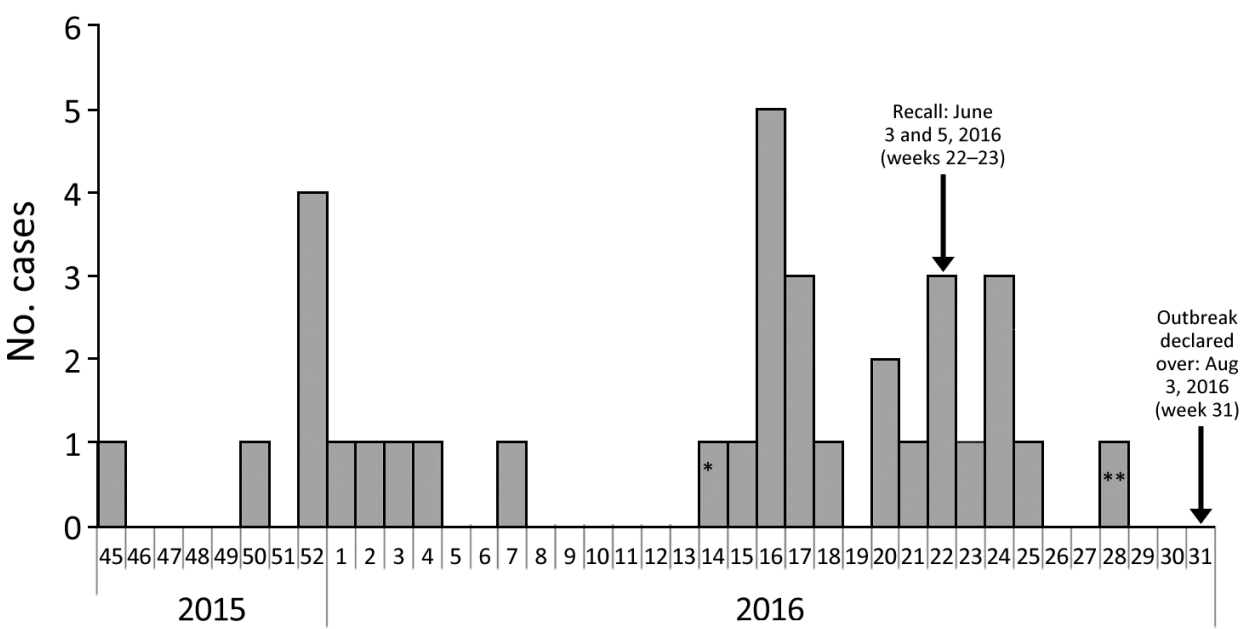

Onset dates bv week and vear 
Although the hypothesis-generating questionnaire used stipulated milk, with flavored milk as a prompt, chocolate milk was not specified, and as a result this type of milk might have been underreported. Exposure to pasteurized milk was reported by $60 \%$ of case-patients in the first wave compared with $76 \%$ of controls. Thus, milk was not originally pursued as a source. However, this new positive isolate led to reinterviewing of case-patients from the second wave and resulted in $9(75 \%)$ of 12 case-patients reporting consuming brand B when asked specifically about chocolate milk.

On June 3, a retail sample of brand B chocolate milk produced at facility $\mathrm{C}$ was confirmed positive for $L$. monocytogenes. This finding led to a class I recall of 1 lot of brand B chocolate milk. On June 5, the recall was expanded to all lots of brand B chocolate milk processed at that facility that only distributes in Ontario. Isolates from the original sample and 3 subsequent positive samples of chocolate milk, obtained from extensive retail sampling, matched the outbreak strain by PFGE and whole-genome sequencing. No white milk samples were positive for $L$. monocytogenes.

Environmental sampling at the manufacturer confirmed the presence of the outbreak strain within a postpasteurization pump dedicated to chocolate milk and on nonfood contact surfaces. This postprocess contamination of the chocolate milk line was believed to be the root cause of the outbreak. A harborage site might have been introduced by a specific maintenance event or poor equipment design. The equipment was subsequently replaced, and corrective measures were implemented to prevent recurrence. Chocolate milk production was resumed after rigorous testing for L. monocytogenes under regulatory oversight.

\section{Conclusions}

This outbreak lasted 7 months and resulted in 34 confirmed listeriosis case-patients. Discovering the cause of this listeriosis outbreak was challenging because pasteurized chocolate milk is a commonly consumed product. Although there have been previous outbreaks outside Canada caused by chocolate milk (8), pasteurized milk products are generally not expected to be the source. This outbreak highlights that even pasteurized products can be contaminated by and support the proliferation of L. monocytogenes when contamination is introduced postpasteurization. The possibility of postprocessing contamination indicates an ongoing need for regulatory oversight and robust quality assurance processes, which include routine sampling of the environment and finished products.

Brand B chocolate milk is a widely distributed product in Ontario, and contamination of this product could have resulted in $>34$ case-patients. It is possible that a lower number of case-patients were reported because chocolate milk may primarily be consumed by younger, healthier persons, in whom invasive listeriosis is less likely to develop (9). Another possible explanation is that the contamination in the milk

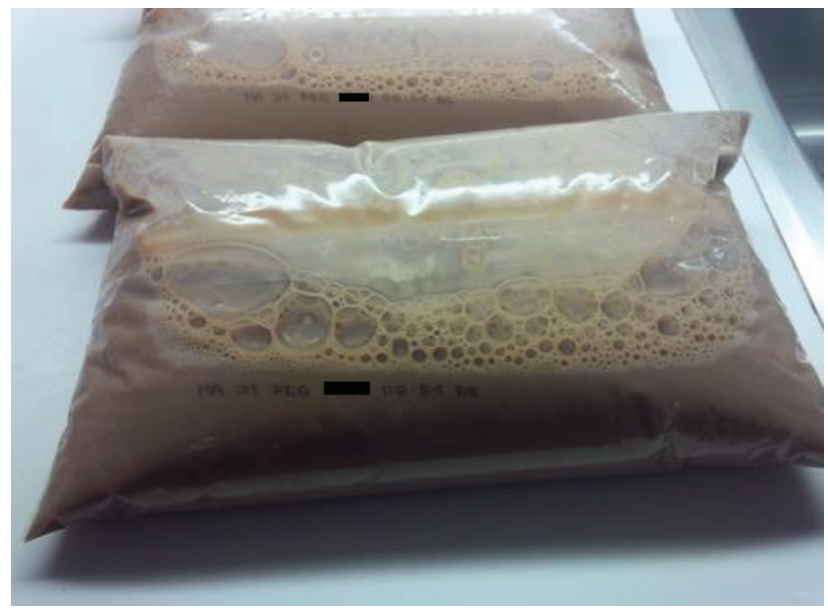

Figure 2. Bags of pasteurized chocolate milk as sold in Canada, with outer bag containing brand information removed. A bag of milk similar to these, found at the home of 1 case-patient during investigation of an outbreak of Listeria monocytogenes infection associated with pasteurized chocolate milk in Ontario, Canada, was found to be contaminated with the same strain obtained from infected patients.

appeared to be intermittent, with some samples testing positive and others testing negative. As such, careful attention should be given to equipment design and maintenance programs, as harborage sites could result in recurring contamination that goes undetected by routine monitoring. Targeted retail and environmental sampling was instrumental in identifying the root cause in the facility and the breadth of potentially implicated products in the marketplace. Thus, this type of sampling should be considered during outbreak investigations.

Ultimately, the implicated product was determined on the basis of testing of food items obtained from the home of 1 case-patient. This finding highlights the necessity of obtaining a thorough food history and collecting and testing available samples of food that case-patients consumed during the incubation period (10). In Canada, where bagged milk is common, labeling of the inner and outer bags with the brand name would facilitate product identification by consumers. This recommendation could extend to other food products in North America (e.g., frozen hamburger patties) that have multiple layers of packaging (11).

\section{Acknowledgments}

We thank Public Health Ontario, Public Health Ontario Laboratory, Ontario Ministry of Health and Long-Term Care, Public Health Agency of Canada, Ontario Ministry of Agriculture and Rural Affairs, Canadian Food Inspection Agency, National Microbiology Laboratory, Durham Region Health Department, Middlesex-London Health Unit, Peel Public Health,

Peterborough County-City, Simcoe-Muskoka District Health Unit, Toronto Public Health, Wellington-Dufferin-Guelph Public Health, Windsor-Essex County Health Unit, and York Region Public Health for their participation in this study. 


\section{About the Author}

Ms. Hanson is a senior program specialist in communicable diseases at Public Health Ontario, Toronto, Ontario, Canada. Her research interests include foodborne disease surveillance, outbreak investigations, risk factors for infectious diseases, and health equity.

\section{References}

1. Ontario Agency for Health Protection and Promotion (Public Health Ontario). Reportable disease trends in Ontario: listeriosis. Toronto: Queen's Printer for Ontario; 2017.

2. Currie A, Farber JM, Nadon C, Sharma D, Whitfield Y, Gaulin C, et al. Multi-province listeriosis outbreak linked to contaminated deli meat consumed primarily in institutional settings, Canada, 2008. Foodborne Pathog Dis. 2015;12:645-52. http://dx.doi.org/ 10.1089/fpd.2015.1939

3. Gaulin C, Ramsay D, Bekal S. Widespread listeriosis outbreak attributable to pasteurized cheese, which led to extensive cross-contamination affecting cheese retailers, Quebec, Canada, 2008. J Food Prot. 2012;75:71-8. http://dx.doi.org/10.4315/ 0362-028X.JFP-11-236

4. Heymann DL, editor. Control of communicable diseases manual 20th ed. Washington (DC): American Public Health Association; 2015 [cited 2018 Dec 14]. https://www.apha.org/ccdm

5. Newkirk R, Hedberg C, Bender J. Establishing a milkborne disease outbreak profile: potential food defense implications. Foodborne Pathog Dis. 2011;8:433-7. http://dx.doi.org/10.1089/ fpd.2010.0731

6. Public Health Agency of Canada. Outbreak of Listeria infections linked to packaged salad products produced at the Dole processing facility in Springfield, Ohio, 2016 [cited 2018 Dec 17]. https://www.canada.ca/en/public-health/services/public-healthnotices/2016/public-health-notice-update-outbreak-listeriainfections-linked-packaged-salad-products-produced-doleprocessing-facility-springfield-ohio.html

7. Centers for Disease Control and Prevention. Multistate outbreak of listeriosis linked to packaged salads produced at Springfield, Ohio Dole processing facility, 2016 [cited 2018 Dec 17]. https://www.cdc.gov/listeria/outbreaks/bagged-salads-01-16/ index.html

8. Dalton CB, Austin CC, Sobel J, Hayes PS, Bibb WF, Graves LM, et al. An outbreak of gastroenteritis and fever due to Listeria monocytogenes in milk. N Engl J Med. 1997;336:100-5. http://dx.doi.org/10.1056/NEJM199701093360204

9. Sebastian R, Goldman J, Wilkinson EC, LaComb R. Fluid milk consumption in the United States: what we eat in America, NHANES 2005-2006. Beltsville (MD): US Department Of Agriculture, Agricultural Research Service, Beltsville Human Nutrition Research Center; 2010 [cited 2018 Dec 14]. https://www.ars.usda.gov/ARSUserFiles/80400530/pdf/DBrief/ 3_milk_consumption_0506.pdf

10. Centers for Disease Control and Prevention. Outbreak of Listeria monocytogenes infections associated with pasteurized milk from a local dairy-Massachusetts, 2007. MMWR Morb Mortal Wkly Rep. 2008;57:1097-100.

11. Morton V, Whitfield Y, Cutler J, MacDonald D. Frozen beef hamburgers associated with two E. coli $\mathrm{O} 157$ outbreaks in Canada, 2012-2013. Environmental Health Review. 2017; 60:28-33.

Address for correspondence: Heather Hanson, Public Health Ontario, 480 University Ave, Ste 300, Toronto, ON M5G 1V2, Canada; email: heather.hanson@oahpp.ca

\section{EID SPOTLIGHT TOPIC}

\section{Food Safety}

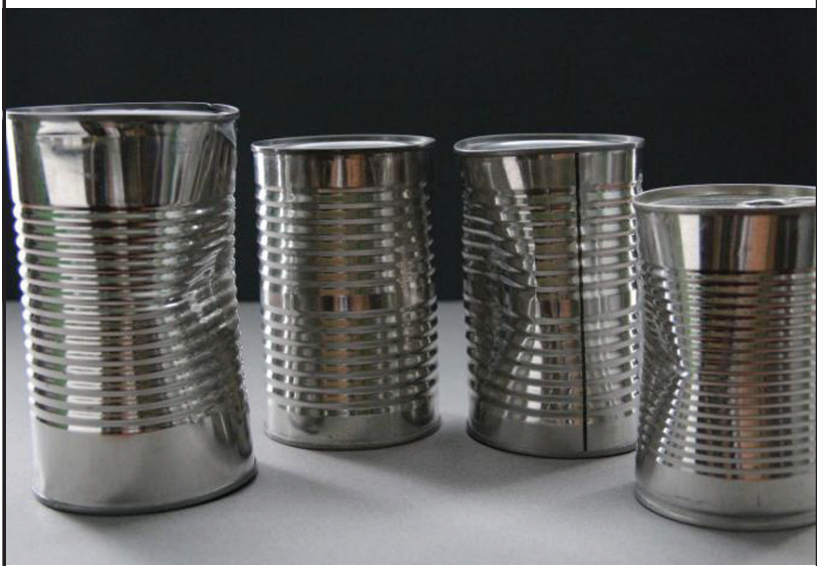

Foodborne illness (sometimes called

"foodborne disease," "foodborne infection," or "food poisoning") is a common, costlyyet preventable-public health problem. Each year, 1 in 6 Americans gets sick by consuming contaminated foods or beverages. Many different disease-causing microbes, or pathogens, can contaminate foods, so there are many different foodborne infections. In addition, poisonous chemicals or other harmful substances can cause foodborne diseases if they are present in food.

\section{Visit our website to listen:} http://wwwnc.cdc.gov/eid/page/ food-safety-spotlight

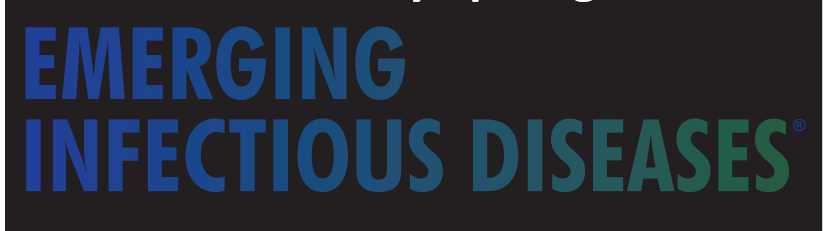

